\title{
Finger Knuckle Identification using RLF and Dynamic Time Warping
}

\author{
Shubhangi Neware \\ Research Scholar \\ Dr. C.V. Raman University \\ Bilaspur (C.G.)
}

\author{
Kamal Mehta, PhD \\ Associate Professor \\ Institute of Technology, Nirma \\ University, Ahmadabad (Gujrat)
}

\author{
A. S. Zadgaonkar, PhD \\ Advisor \\ Dr. C.V. Raman University \\ Bilaspur (C.G.)
}

\begin{abstract}
Texture pattern of finger back surface is highly unique consisting of creases and lines which can be used for biometric authentication system. Use of Finger Knuckle Print (FKP) for person identification has been attracted attention of researchers in last few years .Finger Knuckle Print is becoming emerging biometric identifier. In this paper, we present a finger knuckle identification method that uses Dynamic programming (DP) for the alignment of Radon Like Features. The key idea is to use dynamic time warping (DTW) to match Radon Like features of two knuckle images. Experiment is carried out using IIT Delhi finger knuckle database version 1.0. Knuckle features are extracted using the Radon Like Feature technique is classified using DTW for the identification of finger knuckle print. Result obtained using RLF and DTW is promising as compared to subspace and Gabor filtering methods.
\end{abstract}

\section{Keywords}

Biometrics, Finger Knuckle Print (FKP), Radon Like Feature (RLF), Knuckle, Dynamic Time Warping.

\section{INTRODUCTION}

Finger knuckle print can be highly useful for user identification. FKP as a new biometric modality providing wide scope for researchers. Kumar A et al [2] used PCA, LDA, ICA subspace techniques for feature extraction. The Principal Component Analysis (PCA) determines the basis vectors for spanning an optimal subspace such that the mean square error between the projections of the training images onto this subspace and the original images is reduced. Independent Component Analysis (ICA) is the subspace technique that can create spatially localized features. Linear Discriminant Analysis (LDA) finds vectors in the underlying space that are the best discriminates among classes. Nigam et al [3] extracted knuckle features using scale invariant feature transform (SIFT) and the speeded up robust features (SURF), which are used to extract the local features from a FKP image. Both of these methods determine scale invariant key-points and describe these key-points by means of local patterns around key-points. Kumar et al [7] [15] exploits the orientation features from the random knuckle lines using Finite Radon Transform. Z. Lin [8] applied feature extraction scheme that combines orientation and magnitude information extracted by Gabor filtering. Z Lin [9] applied competitive coding scheme, which uses $2 \mathrm{D}$ Gabor filters to extract the image local orientation information to represent the FKP features. In this paper we proposed FKP feature extraction using Radon like features [19] and feature matching using DTW algorithm. Dynamic Time Warping (DTW) [5] has long been used to find the optimal alignment of two signals.
The DTW algorithm calculates the distance between each possible pair of points out of two signals. DTW can be used to synchronize two feature vectors that are out of phase. Synchronization ensures proper comparison of associated feature values. It uses these distances to calculate a cumulative distance matrix and finds the least expensive path through this matrix. This path represents the ideal warp - the synchronization of the two signals which causes the feature distance between their synchronized points to be minimized. DTW has been used in various fields, such as speech recognition, data mining, and movement recognition [17]. Ahmed H. proposed Dynamic time warping algorithm using Euclidean distances between the features of the two points is used for alignment of the two signatures one dimensional feature vectors [21].Previous work in the field of DTW mainly focused on speeding up the algorithm.

\section{RADON LIKE FEATURES}

The knuckle image mainly consists of curved lines and creases. The Radon transform can effectively accentuate such line features by summation of image pixels along several directions and is highly computationally efficient. The Radon Transform (RT) is a technique used extensively in tomography applications. It is defined by the integrated function $I(x, y)$ on a line $l$ that is characterized by an intercept $m$ and a slope $z$ :

$$
\begin{aligned}
& R(m, z)[I(x, y)] \\
& =\int_{-\infty}^{+\infty} I(x, m+z y) d x \ldots \ldots .
\end{aligned}
$$

Furthermore, for each intercept and slope in space, the Radon back-projection is used to reconstruct the original input image. Kumar et al [10] propose an other approach based on Radon Transform called Radon Like Features (RLF). RLF retains the central idea from the Radon Transform i.e. processing an image ( a 2D function $\mathrm{I}(\mathrm{x}, \mathrm{y})$ ) along a line ( 1 , parameterized by $t$, i.e. $1(t)=(x(t), y(t)))$.But instead of collapsing $\mathrm{I}(\mathrm{x}, \mathrm{y})$ along line 1 into a scalar value via integration ( as in equation 1), RLF distribute some desired information derived from $\mathrm{I}(\mathrm{x}, \mathrm{y})$ among various line segments along 1 . In our paper numbers of lines are thirty six. The line segments can be defined by the set of principal points along line 1 . The set of principal points along line 1 is given as $\left(t_{1}, t_{2} \ldots \ldots . t n\right)$, value of RLF at a point $\mathrm{P}$ along a line 1 between ( $\mathrm{x}(\mathrm{ti}), \mathrm{y}(\mathrm{ti}))$ and $\left(x\left(t_{i+1}\right), y\left(t_{i+1}\right)\right)$ is given by equation- 2 :

$$
\begin{array}{r}
\psi\left(P, l, t_{i}, t_{i+1}\right)[I(x, y)]=T(I, l(t)), \\
t \in\left[t_{i}, t_{i+1}\right] \ldots \ldots \ldots \ldots
\end{array}
$$


In this equation $\mathrm{T}$ can be any desired function called extraction function. Each line has been associated with some direction. Here angle $\theta$ is used in association with each line where $\theta \in(\theta, 2 \pi)$ whose tangent gives its slop. RLF is intended to redistribute the statistical input image in the compact feature descriptors and to improve the cell boundary from image. In this paper knuckle images are enhanced using RLF. The main idea behind the use RLF in knuckle image is to enhance the line and creases in an image. Knuckle image can be enhanced using Radon Like Features as shown in figure2.

\section{LINE FEATURE EXTRACTION USING RLF}

RLF of knuckle images are computed using thirty six line segments. For experiment publicly available "IIT Delhi finger knuckle database version 1.0" [11] is used. This database has been acquired from 158 users in IIT Delhi campus using digital camera .All the subjects in the database are in the age group 16 to 55 years. Images are in bitmap format. Resolution of these images is $80 \times 100$ pixels. Sample knuckle images are as shown in figure 1(a). Figure 1(b) represents the enhanced Knuckle images using RLF technique. It has been observed that edges are more visible in Figure 2 as compared to original image.

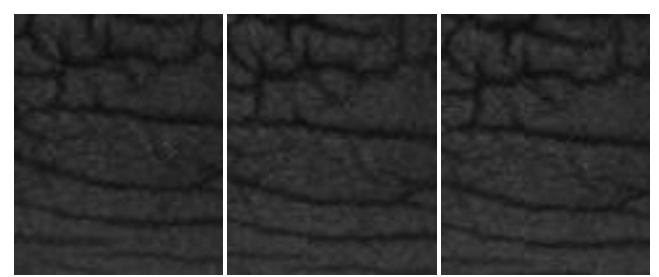

Figure 1 (a): Finger Knuckle Image sample

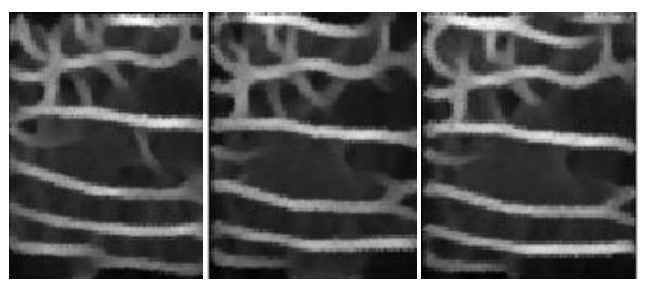

Figure 1(b): Enhanced knuckle images using RLF

\section{DYNAMIC TIME WARPING}

The dynamic time warping is an algorithm to calculate the optimal score and to find the optimal alignment between two strings. This method is a much more robust distance measure for time series than Euclidean distance, allowing similar shapes to match even if they are out of phase in the time axis [18]. Any distance method such as Euclidean which aligns the $i$-th point on one time series with the $i$-th point on the other will produce a poor similarity score. A non-linear (elastic) alignment produces a more intuitive similarity measure, allowing similar shapes to match even if they are out of phase in the time axis as shown in figure 2[20].

Originally, DTW has been used to compare different speech patterns in automatic speech recognition. In fields such as data mining and information retrieval, DTW has been successfully applied to automatically cope with time deformations and different speeds associated with time-dependent data.

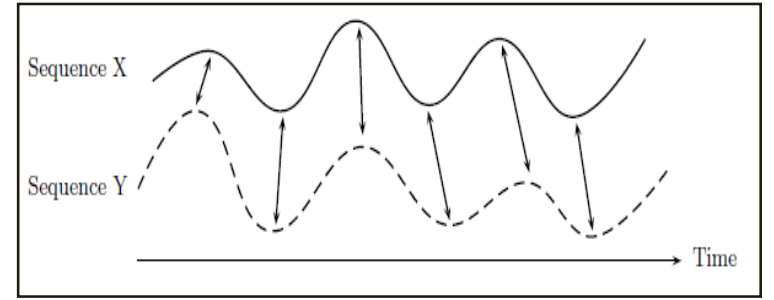

Figure 2 : Mapping of two feature vectors using DTW

\subsection{FKP Matching using Dynamic Time Warping}

When determining the DTW-distance $\operatorname{dist}(\mathrm{X}, \mathrm{Y})$ between two Knuckle images

$X=\left(x_{1}, x_{2}, \ldots \ldots \ldots, x_{M}\right)$
$Y=\left(y_{1}, y_{2}, \ldots \ldots \ldots, y_{N}\right)$

a matrix $D$ of size $M \times N$ is built, where each entry $D(i, j)(1 \leq$ $\mathrm{i} \leq \mathrm{M}, 1 \leq \mathrm{j} \leq \mathrm{N})$ is the cost of aligning the subsequences $\mathrm{X} 1$ : $\mathrm{i}$ and $Y 1: j$. Each entry $D(i, j)$ is calculated from some $D\left(i^{\prime}, j^{\prime}\right)$ plus an additional cost $d$, which is usually some distance between the samples xi and yi [16]. i.e. To calculate the DTW distance $\mathrm{D}(\mathrm{X}, \mathrm{Y})$, we can first construct an $\mathrm{M} \times \mathrm{N}$ matrix, as shown in fig. Then, we find a path in the matrix which starts from cell (1:1) to cell (M:N) so that the average cumulative cost along the path can be minimized as shown in figure 3(b). Path indicates the optimal alignment. [21].

$\mathrm{D}(\mathrm{i}, \mathrm{j})$ is a minimal distance and is given as

$$
D(i, j)=\min \left\{\begin{array}{c}
D(i, j-1) \\
D(i-1, j) \\
D(i-1, j-1)
\end{array}\right\}+d\left(x_{i}, y_{j}\right)
$$

The recursive definition of $\mathrm{D}(\mathrm{i}, \mathrm{j})$ based on the given three values is a local continuity constraint $D(i, j-1), D(i-$ $1, j)$ and $D(i-1, j-1)$ as shown in figure $3(\mathrm{a})$.

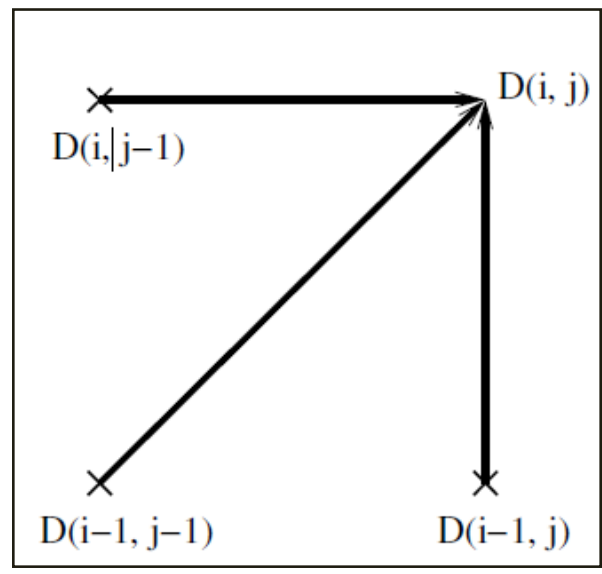

Figure 3(a): Definition of $D(i, j)$ 


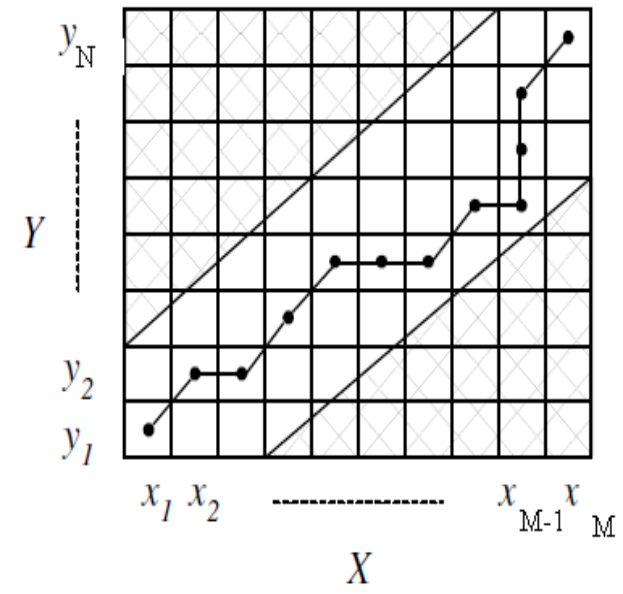

Figure 3(b): Minimal path obtained by DTW

\section{DTW algorithm :}

Input:

$X=\left(x_{1}, x_{2}, \ldots \ldots, x_{M}\right)$

$Y=\left(y_{1}, y_{2}, \ldots \ldots, y_{N}\right)$

Distance function $\mathrm{d}($ )

Output: DTW matrix D $(\mathrm{M} \times \mathrm{N})$

Algorithm:

Step 1: $D(1,1)=d\left(x_{1}, y_{1}\right)$;

Step 2: for $m=1$ to $M$

$$
D(m, 1)=D(m-1,1)+d\left(x_{m}, y_{1}\right) ;
$$

Step 3: for $\mathrm{n}=1$ to $\mathrm{N}$

$$
D(1, n)=D(1, n-1)+d\left(x_{1}, y_{n}\right)
$$

Step 4: for $\mathrm{i}=2$ to $\mathrm{M}$

$$
\text { for } \mathrm{j}=2 \text { to } \mathrm{N}
$$

$$
D(i, j)=\min \left\{\begin{array}{c}
D(i, j-1) \\
D(i-1, j) \\
D(i-1, j-1)
\end{array}\right\}+d\left(x_{i}, y_{j}\right)
$$

\section{EXPERIMENTAL RESULTS}

Experimental result using "IIT Delhi finger knuckle database version 1.0 " is as shown in figure 4 and 5.

\begin{tabular}{l} 
Input images: D: $\backslash$ shubhu $\backslash 003$ 5.bmp \\
Recognized ID: 3 \\
----------------Distance---------------------------- \\
2.9754 e+003 \\
\\
\hline \hline Start Busy
\end{tabular}

Figure 4 : Result of DTW matching showing recognized ID and matching distance.

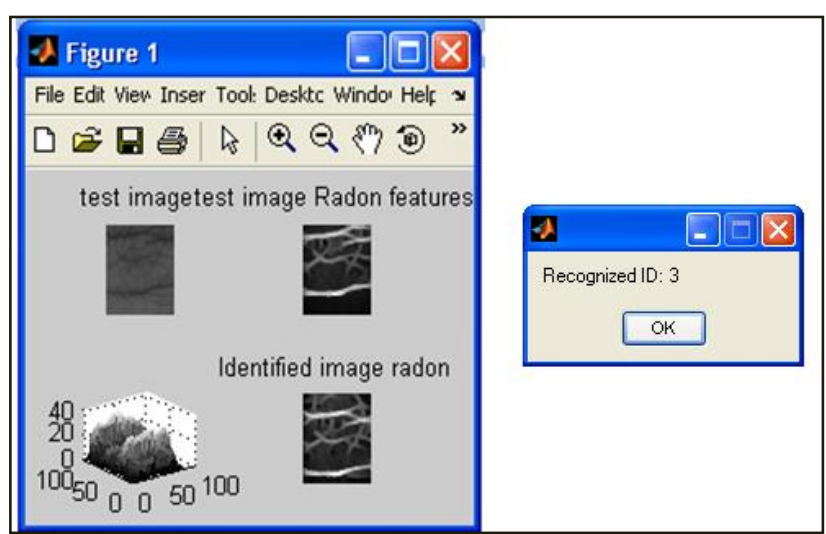

Figure 5: RLF of Test image and Identified knuckle image.

The performance of the biometrics-based verification system is evaluated by the Receiver Operating Characteristic (ROC) curve as shown in figure 6, which illustrates the False Rejection Rate (FRR) against the False Acceptance Rate (FAR) at different thresholds on the matching score. We first evaluate the FRR for all the possible combinations of genuine attempts. Next, we evaluate the FAR for all the possible combinations of impostor attempts. The performance is also evaluated by the Equal Error Rate (EER), which is defined as the error rate where the FRR and the FAR are equal. From graph EER is observed as 0.17. Table 1 shows the values of FAR,FRR and 1-FRR for different threshold values of matching distance.

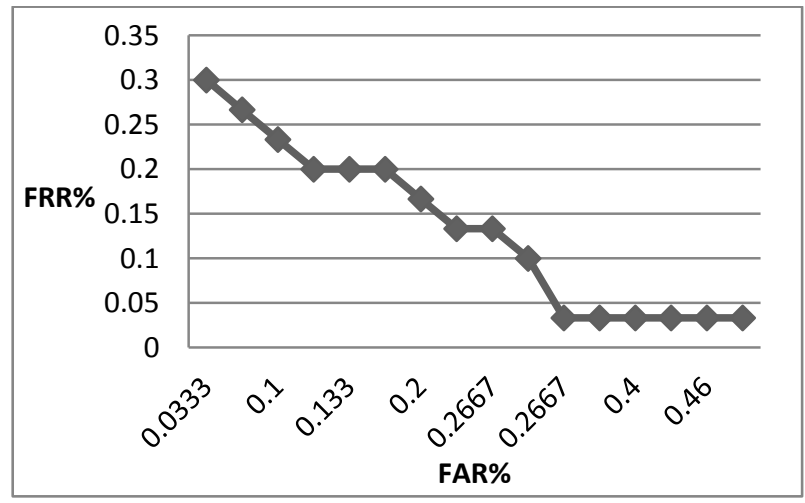

Figure 6 : ROC curve using DTW matching method 
Table1: Typical operating states for different threshold value

\begin{tabular}{|l|l|l|l|}
\hline Threshold & FAR & FRR & 1-FRR \\
\hline 3.6 & 0.0333 & 0.3 & 0.7 \\
\hline 3.7 & 0.0333 & 0.266 & 0.74 \\
\hline 3.8 & 0.1 & 0.233 & 0.77 \\
\hline 3.9 & 0.133 & 0.2 & 0.80 \\
\hline 4.0 & 0.133 & 0.2 & 0.80 \\
\hline 4.1 & 0.133 & 0.2 & 0.80 \\
\hline 4.2 & 0.2 & 0.16667 & 0.84 \\
\hline 4.3 & 0.266 & 0.13333 & 0.87 \\
\hline 4.4 & 0.266 & 0.13333 & 0.87 \\
\hline 4.5 & 0.266 & 0.1 & 0.90 \\
\hline 4.6 & 0.266 & 0.03333 & 0.97 \\
\hline 4.7 & 0.333 & 0.03333 & 0.97 \\
\hline 4.8 & 0.4 & 0.03333 & 0.97 \\
\hline 4.9 & 0.4 & 0.03333 & 0.97 \\
\hline 5.0 & 0.46 & 0.03333 & 0.97 \\
\hline 5.1 & 0.56 & 0.03333 & 0.97 \\
\hline
\end{tabular}

\section{CONCLUSION}

In this paper Radon Like Feature (RLF) is applied for feature extraction of two dimensional finger knuckle images .It is more effective method as compared to simple Radon Transform as features has been extracted along multiple scan lines with different angles. Also RLF method is suitable for the enhancement of knuckle images that contains lines or creases. Extracted knuckle features using RLF method is then used for person identification purpose. In this work Dynamic Time Warping (DTW) technique is used to match knuckle images. DTW allows a non-linear mapping of one feature image to another by minimizing the distance between the two. It has been observed that DTW is producing better results with reduced EER as compared to other methods.

\section{REFERENCES}

[1] Jain, A.K., Flynn, P., Ross, A. (eds.): Handbook of Biometrics. Springer, Heidelberg(2007)

[2] Kumar A and Ravikanth C, "Personal authentication using finger knuckle surface", IEEE Transactions on Information Forensics and Security, 4(1):98 -110, 2009.

[3] Badrinath G S, Nigam A. and Gupta P, "An Efficient Finger-knuckle-print based Recognition System Fusing SIFT and SURF Matching Scores", Information and communication Security, pp374-387, 2011.

[4] Woodard D.L., Flynn P.J., "Finger surface as a biometric identifier", CVIU, vol. 100, pp. 357-384, 2005.

[5] Neware S, Mehta K, Zadgaonkar A, "Finger Knuckle Identification using Principal Component Analysis and Nearest Mean Classifier", International Journal of Computer Applications (0975 - 8887) Volume 70- No.9, May 2013

[6] Neware S, Mehta K, Zadgaonkar, "Finger Knuckle Surface Biometrics", International Journal of Emerging Technology and Advanced Engineering ,ISSN 22502459, Volume 2, Issue 12, December 2012.
[7] Neware S, Mehta K, Zadgaonkar, "Finger Knuckle Print Identification using Gabor Features", International Journal of Computer Applications (0975 - 8887) Volume 98- No.16, July 2014

[8] Kumar A and Zhou Y, "Human identification using knuckle codes", Proceedings BTAS, Washington, 2009.

[9] Zhang L, Zhang L, Zhang D, Zhu H, "Online fingerknuckle-print verification for personal authentication",Pattern Recognition, 43(7):2560-2571, Elsevier, July 2010.

[10] Zhang L, Zhang L, Zhang D, "Finger-Knuckle Print: A New Biometric Identifier", Image Processing ICIP, IEEE International Conference, pp1981-84, Nov2009.

[11] Kumar R, V'azquez-Reina A, Pfister H "Radon-Like Features and their Application to Connectomics" ,IEEE Workshop on Mathematical Methods in Biomedical Image Analysis (MMBIA10), San Francisco, CA, June 15-17, 2010.

[12] IIT Delhi Finger Knuckle Database (Version 1.0) http://www4.comp.polyu.edu.hk/ CSajaykr/IITD/iitd_kn uckle.htm

[13] Celik T, "Automatic Image Equalization \& Contrast Enhancement using Gaussian Mixture Modeling ",Image Processing ,IEEE Transactions,Vol-21 Issue 1 July 2011.

[14] Woodard D.L., Flynn P.J., "Finger surface as a biometric identifier", CVIU, vol. 100, pp. 357-384, 2005.

[15] Shoichiro A, Koichi I, Takafumi A, "Finger-KnucklePrint Recognition Using BLPOC-Based Local Block Matching”, IEEE, 2011.

[16] Kumar A, Zhou Y, "Personal Identification using Finger Knuckle Orientation Features", Electronics Letters ,vol 45, no. 20, September 2009.

[17] Santosh K.C., Bart Lamiroy, Laurent Wendling. DTWRadon-based Shape Descriptor for Pattern Recognition. International Journal of Pattern Recognition and Arti_cial Intelligence, World Scienti_c Publishing, 2013, $27(3)$

[18] Toni Giorgino, "Computing and Visualizing Dynamic Time Warping Alignments in R: The dtw Package", Journal of Statistical Software, August 2009, Volume 31, Issue $7 .$.

[19] E. Keogh "Exact indexing of dynamic time warping", Proceeding of the 28th VLDB Conference, 2002.

[20] Mehta K, Neware S, Zadgaonkar A , "Finger Knuckle Feature Extraction using Radon like Features", IJCSC , ISSN 0973-7391, pp.134-137, July-Sep 2014

[21] Muller M ,'Information Retrieval for Music and Motion",Springer,ISBN:978-3-540-74047-6, XVI,318p,2007

[22] Ahmed H, Shukla S, "Comparative Analysis of Global Feature Extraction Methods for Off-line Signature Recognition", International Journal of Computer Applications (0975 - 8887) Volume 48- No.23, June 2012 